\title{
Correction to: Evaluating the Effect of a Video Education Curriculum for First Time Breast Cancer Patients: a Prospective RCT Feasibility Study
}

\author{
Nika Sulakvelidze ${ }^{1}$ - Brian Burdick ${ }^{1,2,3,4,5,6,7} \cdot$ Eric Gelfand $^{1,2,3,4,5,6,7} \cdot$ Virginia Kaklamani $^{2} \cdot$ Kay Tilton $^{3} \cdot$ Kelsey Baker $^{4}$. \\ Janice $\mathrm{Kim}^{5}$ - Sara Javid ${ }^{6}$. Julie R. Gralow ${ }^{7}$
}

Published online: 24 October 2019

(C) American Association for Cancer Education 2019

\section{Correction to: Journal of Cancer Education}

https://doi.org/10.1007/s13187-019-01578-3

The original version of this article unfortunately contained a mistake. The name of "Eric Gelfand" was omitted.

Publisher's Note Springer Nature remains neutral with regard to jurisdictional claims in published maps and institutional affiliations.

The online version of the original article can be found at https://doi.org/ 10.1007/s13187-019-01578-3

Nika Sulakvelidze

nsulakvelidze@gmail.com

1 University of Washington School of Medicine, Seattle WA USA

2 Department of Medicine, Division of Hematology and Oncology, University of Texas Health Science Center - San Antonio, San Antonio TX USA

3 Division of Breast Oncology, Seattle Cancer Care Alliance, Seattle WA USA

4 Division of Clinical Research, Fred Hutchinson Cancer Research Center, Seattle WA USA

5 Department of Radiation Oncology, University of Washington School of Medicine, Seattle WA USA

6 Department of Surgery, Division of Oncology, University of Washington School of Medicine, Seattle WA USA

7 Department of Medicine, Division of Medical Oncology, University of Washington School of Medicine, Seattle WA USA 